\title{
Eficacia de una intervención educativa sobre conocimientos de ley chilena de SIDA en trabajadores de salud primaria
}

\author{
LILIAN FERRER ${ }^{1,2,3, a}$, BALTICA CABIESES ${ }^{1, a}$, KATHLEEN NORR $^{2, \mathrm{~b}}$, \\ ROSINA CIANELLI ${ }^{1,3, a}$, ALEJANDRA ARAYA ${ }^{1, a}$, LISETTE IRARRÁZABAL ${ }^{1, a}$, \\ SARAH MINER ${ }^{1, \mathrm{c}}$, MARGARITA BERNALES $^{1, \mathrm{~d}}, \mathrm{JAMES} \mathrm{NORR}^{2, \mathrm{~b}}$
}

\section{Effectiveness of an educational program about the Chilean AIDS law in primary care health workers}

Background: In Chile, members of the civil society and government achieved the passing of the HIV/AIDS Law (19.779). The level of knowledge of the law held by healthcare workers in Chile is not well known. Aim: To analyze the effect of an intervention on knowledge of the existence of the law and its application in clinical practice among primary healthcare workers in southeastern Santiago. Material and Methods: Healthcare workers of primary care centers were invited to participate in the study. One group received an educational intervention lasting a total of 16 hours, about AIDS physiopathology, sexually transmitted diseases, communication with patients and current legislation. A control group did not receive the educational intervention. Both groups answered a self-administered questionnaire about the HIV/AIDS law at baseline and three months after the intervention. Results: The intervention was carried out in 262 workers and 293 participated as controls. The initial evaluation revealed that only $16.3 \%(n=89)$ had heard of the law, without any significant difference between intervention and control groups. The knowledge about the law improved by $65 \%$ in the intervention group and did not change in controls. At the end of the education period, the intervention and control groups improved their global knowledge by 29 and 3\%, respectively $(p<0.05)$. Conclusions: The educational intervention was effective in improving knowledge of the HIV/AIDS Law among Chilean healthcare workers.

(Rev Med Chile 2011; 139: 625-632).

Key words: Health education; HIV; Patient care team; Primary health care.

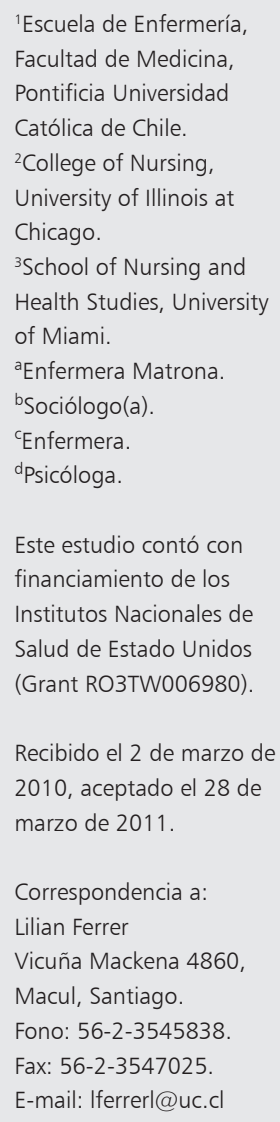

'Escuela de Enfermería, Facultad de Medicina, Pontificia Universidad Católica de Chile. ${ }^{2}$ College of Nursing, University of Illinois at Chicago. ${ }^{3}$ School of Nursing and Health Studies, University of Miami.

aEnfermera Matrona.

bSociólogo(a).

'Enfermera.

'Psicóloga.

Este estudio contó con financiamiento de los Institutos Nacionales de Salud de Estado Unidos (Grant RO3TW006980).

Recibido el 2 de marzo de 2010, aceptado el 28 de marzo de 2011.

Correspondencia a: Lilian Ferrer Vicuña Mackena 4860, Macul, Santiago. Fono: 56-2-3545838. Fax: 56-2-3547025. E-mail: Iferrerl@uc.cl

S egún el último reporte del Programa Conjunto sobre el VIH/SIDA, se estima que viven 33,4 millones de personas con VIH en el mundo ${ }^{1}$. En Chile, se han notificado 22.115 casos de VIH/SIDA desde su primera notificación en 1984 hasta $2009^{2}$.

Desde los inicios de esta epidemia ha existido estigmatización y discriminación hacia las personas que viven con el virus. Se han reportado abusos que atentan contra los derechos humanos hacia personas que viven con VIH (PVVIH), como por ejemplo negación y cuestionamiento del cuidado de salud en el ámbito laboral y educacional, además de maltrato físico y psicológico ${ }^{3-7}$.

En este contexto, la comunidad mundial ha dispuesto de una serie de medidas para el control de la epidemia y la protección de PVVIH, entre las que se encuentra la "Declaración de Compromiso en la lucha contra el VIH y SIDA". El documento plantea la protección legal de PVVIH, para asegurar el cumplimiento de los derechos y libertades humanas fundamentales como el acceso a la edu- 
cación, empleo y atención de salud, y respetando la intimidad y confidencialidad de la persona ${ }^{8,9}$.

En Chile, diversas organizaciones de personas viviendo con VIH consensuaron un proyecto de ley $^{10}$, que finalmente derivó en la promulgación de la Ley 19.779. Esta ley de SIDA está centrada en la igualdad de derechos sin discriminación de ninguna índole, y considera la participación de la sociedad civil en la elaboración, implementación y evaluación de políticas Estatales respecto al VIH y SIDA. Los derechos que considera esta ley son: confidencialidad, voluntariedad, consentimiento libre e informado, trabajo, educación y atención en salud ${ }^{11,12}$.

Pese a los avances existentes con la entrada en vigencia de esta ley, aún existen dudas acerca del conocimiento y cumplimiento de ésta por parte de los trabajadores de la salud. Vidal, Carrasco y Santana lo indicaron al comunicar que un gran número de PVVIH en Chile presenta opiniones desfavorables sobre la calidad de atención en salud. Destacaron la ausencia de confidencialidad de la información sobre la condición serológica, la exageración en la utilización de las precauciones estándares, la falta de asertividad de los profesionales encargados de transmitir el diagnóstico, la falta de privacidad en las consejerías y toma del examen, y la realización de procedimientos invasivos sin consentimiento del usuario ${ }^{13}$. Estas situaciones acentúan el estigma percibido por las PVVIH, pudiendo disminuir el acceso a los servicios de salud necesarios, esencial para mantener la salud, el bienestar y la calidad de vida de las personas afectadas por el virus ${ }^{14}$.

Pese a las actitudes y conductas de discriminación reportadas hacia PVVIH, no se ha explorado el nivel de conocimientos que existe sobre la ley 19.779 por parte de trabajadores de la salud en Chile. El objetivo de este estudio fue analizar si una intervención en prevención de VIH y SIDA en trabajadores de salud de atención primaria del sector sur oriente de Santiago mejora los conocimientos en torno a la existencia y normativa de cumplimiento de la ley 19.779 de SIDA.

\section{Metodología}

\section{Diseño}

Se realizó un estudio cuasi-experimental financiado por los Institutos Nacionales de Salud de Estados Unidos de Norteamérica, titulado "Movili- zando trabajadores de la salud para la prevención de VIH/SIDA en Chile" (RO3TW006980) ${ }^{15}$. Esta investigación se llevó a cabo por los proyectos Mano a Mano, una Iniciativa de Investigación en Prevención de VIH/SIDA, durante los años 2004-2007.

\section{Muestra y reclutamiento}

El estudio se realizó en las comunas de Puente Alto y La Pintana. Estas comunas fueron selecionadas por su adecuada representación del sector sur oriente ${ }^{15}$. La definición de las comunas como intervención o control fue aleatoria, siendo La Pintana grupo intervención y Puente Alto grupo control.

El marco muestral fueron los trabajadores de salud de 10 Centros de Salud Primaria de las comunas consideradas para el estudio $(\mathrm{n}=2.100)^{15}$. Se consideró como trabajador de salud a "toda persona comprometida en acciones cuya intención primaria es mejorar la salud" 16 .

Se invitó a participar a la totalidad de trabajadores de salud de los centros que cumplieran con contrato mayor o igual a media jornada laboral de las comunas de la Pintana y Puente Alto. El reclutamiento se realizó mediante carta formal personalizada con autorización del director de cada centro y del alcalde de cada comuna.

La muestra se compuso inicialmente de 720 trabajadores de la salud (364 grupo intervención y 356 grupo control) quienes aceptaron voluntariamente participar. No aceptó participar el $26,8 \%$ $(\mathrm{n}=264)$, los principales motivos fueron licencias prolongadas por Salud Mental o Pre-postnatal y cambios de lugar de trabajo a otra comuna o ciudad. La muestra fue seguida durante tres meses, con una pérdida de 22,9\% $(\mathrm{n}=165)$. El tamaño muestral final considerado para el análisis fue de 555 sujetos (262 grupo intervención y 293 grupo control, Tabla 1), con un poder superior a $80 \%$ para encontrar un incremento igual o superior a $10 \%$ en la escala de conocimientos de Ley de SIDA entre los grupos intervención y control posterior a la capacitación educativa (Programa G Power).

\section{Recolección de datos}

Todos los participantes firmaron un formulario de consentimiento informado. Se utilizó un cuestionario adaptado culturalmente de un proyecto de prevención de VIH y SIDA realizado en África (Grant NR08058) ${ }^{17}$. Dicha adaptación del cuestionario consideró aspectos de la ley de 
Tabla 1. Descripción de flujo de participantes del estudio

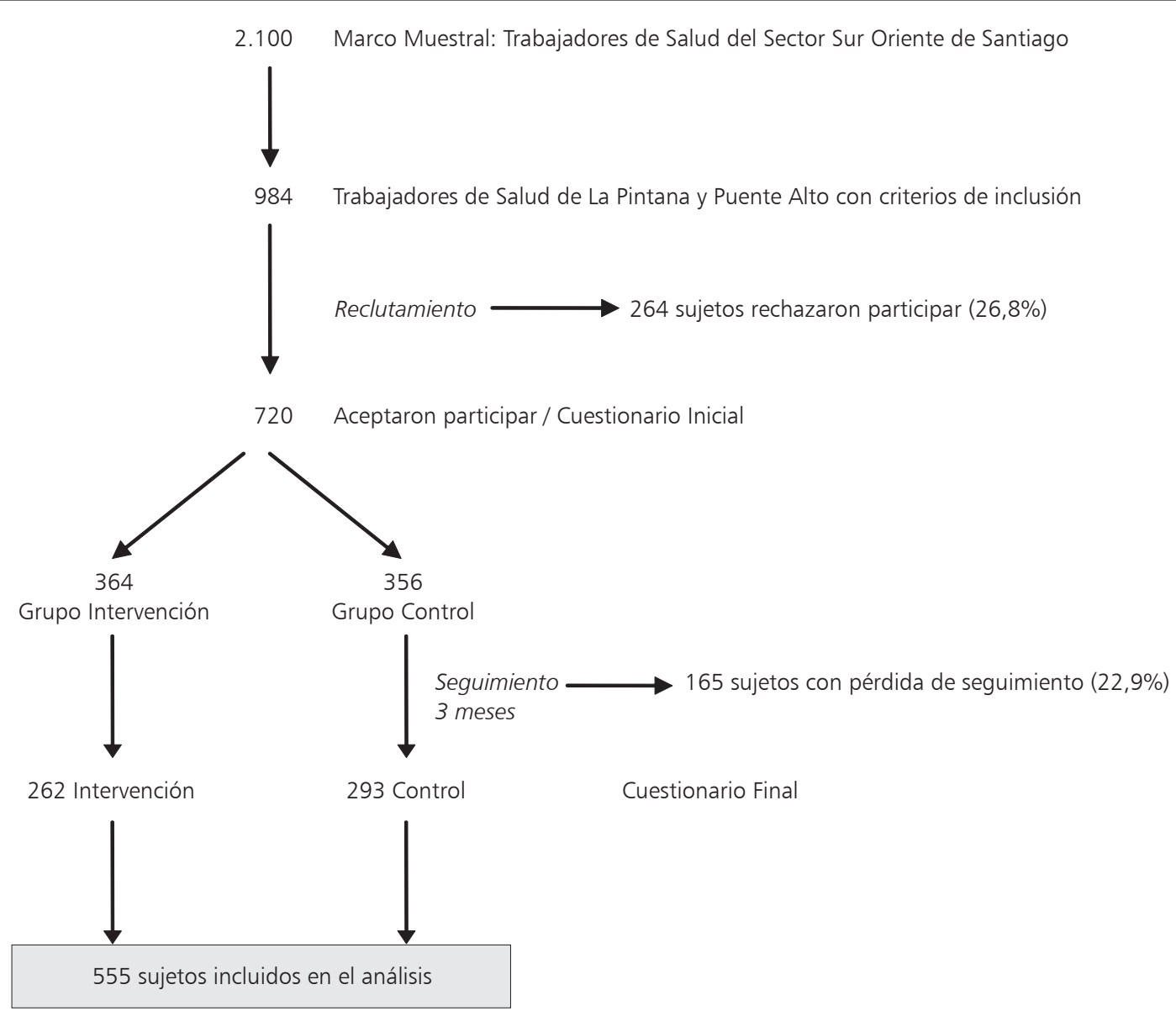

SIDA, que no formó parte del estudio africano, y contó con asesoría de un panel experto en el tema.

Todos los participantes respondieron al cuestionario auto-administrado en dos momentos: al entrar al estudio y luego de tres meses de seguimiento. El cuestionario fue entregado en un sobre cerrado a cada participante del estudio y tuvo una duración de 30-45 minutos. Se pudo responder de inmediato o durante los siguientes 7 días. Cada semana se retiraron los cuestionarios completados de cada centro de salud en sobres cerrados numerados. Se realizó además seguimiento de cuestionarios incompletos por medio de contacto personal o telefónico.

En el período comprendido entre ambas evaluaciones, el grupo intervención recibió una capa- citación desarrollada por el equipo de investigación, previamente entrenado. La estrategia de enmascaramiento del grupo asignado y de participantes no fue considerada en este estudio, sin embargo, el análisis de información fue ciego respecto de la asignación a grupo intervenido o control.

\section{Intervención}

La intervención consistió en 8 sesiones de 2 horas cada una e incluyó los siguientes contenidos: importancia de este fenómeno de salud en Chile, antecedentes fisiopatológicos del VIH y SIDA, normas de precaución estándar, implicancias legales y normativas vigentes en Chile, infecciones de transmisión sexual, comunicación trabajador de salud-usuario, consejería e intervención en crisis. 
La intervención se realizó de acuerdo a los contenidos que emergieron de la primera fase cualitativa del estudio (resultados que no se reportan en este artículo). Los participantes fueron transportados desde sus lugares de trabajo a la Escuela de Enfermería de la Pontificia Universidad Católica de Chile donde se realizó la capacitación a lo largo de 4 semanas (2 sesiones semanales) en grupos pequeños ( $8-12$ personas) y en modalidad de clases participativas y talleres. Esta modalidad consideró una sesión completa de Ley de SIDA. Para esta sesión en particular, se contó con apoyo de un panel de expertos en el tema.

\section{Variables del estudio}

\section{Outcome primario: Score de conocimiento} de Ley de SIDA

Aspectos legales asociados a conocimientos fueron medidos utilizando preguntas desarrolladas por un panel de expertos en el área. Estos ítems consistían en determinar conocimiento sobre Ley de SIDA número 19.779 y sus artículos. Los participantes respondieron a 9 preguntas, las cuales fueron utilizadas como índice (score). Frente a cada afirmación, el participante debía responder: Verdadero, Falso, o No sabe. Posteriormente, se calculó un puntaje total donde la respuesta correcta aportaba un punto y las incorrectas o desconocimiento aportaban cero puntos. El puntaje final alcanzado se distribuía entre 0-9 puntos, donde a mayor puntaje mejor conocimiento (alfa de Cronbach del score de 0,78).

\section{Outcome secundario: Existencia de Ley de SIDA}

Se incluyó una pregunta específica que permitió evaluar si los participantes conocían o no la Ley de SIDA.

\section{Variables de control}

Edad, sexo, nivel de educacional, ingreso económico mensual y tipo de ocupación en el Centro de Salud.

\section{Análisis de datos}

Análisis de estadísticos descriptivos (medidas de tendencia central y de dispersión) se utilizaron para caracterizar a la muestra en relación a variables sociodemográficas y variables principales del estudio. Para el análisis estratificado y comparación pre y post intervención se utilizó prueba de $\chi^{2}$ o T test según tipo de variable. Para el ajuste multivariable se utilizó regresión lineal múltiple, estimándose el coeficiente beta y su significación estadística de la asignación a grupo intervención en presencia de una serie de covariables (nivel educacional, tipo de ocupación e ingreso económico mensual). Los datos fueron analizados utilizando el software computacional Statistical Package for the Social Sciences (SPSS) versión 12.0.

\section{Resultados}

\section{Descripción sociodemográfica de la muestra}

Ochenta porciento de la muestra fue de sexo femenino. Treinta y seis como dos porciento de la muestra presentó educación universitaria completa, 31,5\% educación técnica, 29,3\% educación media y el resto educación básica. Respecto del tipo de ocupación, $33,7 \%$ reportó ocupación clínica de apoyo, 32,8\% administrativo, 22,3\% profesional y el restante 11,2\% técnico. El 40,5\% presentó un ingreso económico mensual menor de 300.000 pesos, $22,3 \%$ entre 301.000 y 500.000 pesos y $37,2 \%$ superior a 500.000 pesos mensuales.

Al comparar las variables sociodemográficas de la muestra según grupo asignado (intervención o control) se observaron diferencias significativas por estratos de nivel educacional, tipo de ocupación e ingreso económico mensual. No se observaron diferencias según sexo y edad entre ambos grupos (Tabla 2).

\section{Conocimiento sobre existencia de la Ley de SIDA}

La evaluación inicial de los trabajadores de la salud reportó que sólo 16,3\% ( $\mathrm{n}=89)$ había escuchado alguna vez sobre la Ley de VIH sin encontrarse diferencias significativas entre el grupo intervención y control.

La evaluación final reportó un aumento significativo del conocimiento acerca de la ley para ambos grupos en conjunto, con un incremento de 33\% $(\mathrm{n}=267)$ respecto de la evaluación inicial ( $p<0,001)$. Al estratificar este resultado por grupo intervención y control, se encontró que el grupo intervenido mejoró su conocimiento de la existencia de la ley en $65 \%(\mathrm{p}<0,001)$, sin embargo, el grupo control no mejoró el conocimiento de la existencia de esta ley en forma significativa $(\mathrm{p}>0,05)$. Ambos grupos fueron significativamente distintos en la evaluación final $(\mathrm{p}<0,05)$ (Tabla 3). 
Score de Conocimiento de la Ley de SIDA para aplicación en la práctica clínica

En promedio, la evaluación inicial reportó que $44,7 \%$ de los trabajadores de la salud practicaría en forma correcta la normativa de prevención de VIH. La evaluación final por su parte reportó que $61,8 \%$ de la muestra llevaría a cabo en forma adecuada dicha normativa. Esto corresponde a un incremento del score de la Ley de SIDA para aplicación en la práctica clínica de $17,1 \%$ tras 3 meses de seguimiento ( $\mathrm{p}<0,05)$. Al comparar según grupo asignado, no se encontraron diferencias en el conocimiento de Ley de SIDA al inicio del estudio. No obstante, al finalizar el seguimiento el grupo intervenido aumentó su conocimiento global para aplicación de la ley en $29,2 \%$, versus un incremento de sólo 3,4\% en el grupo control $(\mathrm{p}<0,05)$. Al evaluar la eficacia de la capacitación, se observó que para cada una de las dimensiones evaluadas se obtuvo un aumento significativo del nivel de conocimiento antes y después de la intervención ( $\mathrm{p}<0,05$, Tabla 4).

De la evaluación al inicio, la variable mejor respondida fue la obligación de realizar consejería previa a la toma del test de Elisa para VIH, que a su vez fue la mejor respondida en la evaluación final, con un incremento significativo de 5,4 puntos porcentuales entre ambas evaluaciones. Las dos

Tabla 2. Descripción sociodemográfica de la muestra en estudio según grupo asignado

\begin{tabular}{|c|c|c|c|}
\hline \multirow{2}{*}{\multicolumn{2}{|c|}{ Características (n) }} & \multicolumn{2}{|c|}{ Porcentaje o promedio (ds) } \\
\hline & & Grupo intervención & Grupo control \\
\hline Sexo masculino (444) & & $23,3 \%$ & $17,1 \%$ \\
\hline Edad (555) & & $41,69(10,6)$ & $37,75(9,8)$ \\
\hline $\begin{array}{l}\text { Nivel educacional } \\
\text { Enseñanza Básica } \\
\text { Enseñanza Media } \\
\text { Enseñanza Técnica } \\
\text { Enseñanza Profesional }\end{array}$ & $\begin{array}{c}(503)^{*}: \\
(17) \\
(162) \\
(174) \\
(200)\end{array}$ & $\begin{array}{r}5,8 \% \\
41,2 \% \\
24,6 \% \\
28,5 \%\end{array}$ & $\begin{array}{r}0,7 \% \\
18,8 \% \\
37,5 \% \\
43 \%\end{array}$ \\
\hline $\begin{array}{l}\text { Tipo de Trabajo } \\
\text { Profesional } \\
\text { Técnico } \\
\text { De apoyo clínico } \\
\text { Administrativo }\end{array}$ & $\begin{array}{c}(555)^{*}: \\
(124) \\
(62) \\
(187) \\
(182)\end{array}$ & $\begin{array}{r}17,2 \% \\
9,2 \% \\
33,6 \% \\
40,1 \%\end{array}$ & $\begin{array}{l}27 \% \\
13 \% \\
33,8 \% \\
26,3 \%\end{array}$ \\
\hline $\begin{array}{l}\text { Ingreso mensual } \\
\quad<300.000 \\
\quad 301.000-500.000 \\
\quad>500.000\end{array}$ & $\begin{array}{l}(551)^{*}: \\
(223) \\
(123) \\
(205)\end{array}$ & $\begin{array}{l}55,2 \% \\
17 \% \\
27,8 \%\end{array}$ & $\begin{array}{l}27,4 \% \\
27,1 \% \\
45,5 \%\end{array}$ \\
\hline
\end{tabular}

${ }^{*} p<0,001$, Test de $\chi^{2}$.

Tabla 3. Distribución porcentual de conocimientos sobre la existencia de la Ley de SIDA, por parte de los trabajadores de la salud $(n=555)$

\begin{tabular}{|c|c|c|c|c|c|c|c|c|}
\hline & \multicolumn{4}{|c|}{ Inicial } & \multicolumn{4}{|c|}{ Final } \\
\hline & $\begin{array}{c}\text { Ambos } \\
\text { grupos } \\
\%\end{array}$ & $\begin{array}{c}\text { Inter- } \\
\text { vención } \\
\%\end{array}$ & $\begin{array}{c}\text { Control } \\
\%\end{array}$ & $\mathbf{p}$ & $\begin{array}{c}\text { Ambos } \\
\text { grupos } \\
\%\end{array}$ & $\begin{array}{c}\text { Inter- } \\
\text { vención } \\
\%\end{array}$ & $\begin{array}{c}\text { Control } \\
\%\end{array}$ & $\mathbf{p}$ \\
\hline $\begin{array}{l}\text { Conoce la Ley de } \\
\text { Prevención de } \\
\text { VIH/SIDA (sí) }\end{array}$ & 16,3 & 17,2 & 15.5 & $>0,05$ & $49,3^{*}$ & $85,1^{*}$ & 17,4 & $<0,05$ \\
\hline
\end{tabular}

${ }^{*} p<0,001$ al comparar resultados inicial versus final. 
variables respondidas en forma deficiente al inicio fueron la obligación de comunicar la situación serológica de un joven de 14 años a sus padres (sólo $11,6 \%$ respondió correctamente) y la obligación de advertir de la situación serológica de un usuario antes de realizar un procedimiento $(13,9 \%)$. Ambas dimensiones mejoraron significativamente tras 3 meses de seguimiento $(\mathrm{p}<0,05)$. El aspecto del cumplimiento de la normativa legal que tuvo un mayor incremento al comparar la evaluación inicial y final fue la referida a comunicar a cónyuges resultado del test, con un incremento de 21,5 puntos porcentuales $(\mathrm{p}<0,05$, Tabla 4$)$.

Las dimensiones antes mencionadas fueron agrupadas en un solo score de correctos conocimientos para cumplimiento de la ley en la práctica laboral, con un recorrido de 0 a 9 puntos. Al inicio el conocimiento de Ley de SIDA en ambos grupos era homogéneo $(\mathrm{p}>0,05)$. La evaluación final reportó un incremento significativo del score de conocimiento en ambos grupos, sin embargo, en incremento fue mayor en el grupo intervenido, con 2,6 puntos versus 0,6 del grupo control ( $\mathrm{p}<0,001$, Tabla 5).

Finalmente, pertenecer al grupo intervención explicó en forma significativa el incremento del conocimiento de Ley de SIDA tras el seguimiento del grupo en estudio (Coeficiente beta $=0,453$,

Tabla 4. Distribución porcentual del score de Ley de SIDA y cómo lo aplicaría durante la práctica clínica $(n=555)$

\begin{tabular}{|c|c|c|c|c|c|c|c|c|}
\hline \multirow{2}{*}{$\begin{array}{l}\text { Ítem del store } \\
\text { Obligación de: }\end{array}$} & \multicolumn{4}{|c|}{ Inicial } & \multicolumn{4}{|c|}{ Final } \\
\hline & $\begin{array}{c}\text { Ambos } \\
\text { grupos } \\
\%\end{array}$ & $\begin{array}{c}\text { Inter- } \\
\text { vención } \\
\%\end{array}$ & $\begin{array}{c}\text { Control } \\
\%\end{array}$ & $\mathbf{p}$ & $\begin{array}{c}\text { Ambos } \\
\text { grupos } \\
\%\end{array}$ & $\begin{array}{c}\text { Inter- } \\
\text { vención } \\
\%\end{array}$ & $\begin{array}{c}\text { Control } \\
\%\end{array}$ & $\mathbf{p}$ \\
\hline $\begin{array}{l}\text { Sugerir esterilización a mujer } \\
\text { con VIH/SIDA }\end{array}$ & 47,6 & 43,5 & 51,2 & $>0,05$ & $65,2^{*}$ & $71,9 *$ & $59,2^{*}$ & $>0,05$ \\
\hline Exigir test de Elisa a usuario & 50,9 & 48,7 & 52,9 & $>0,05$ & $71,4^{*}$ & $80,9 *$ & $62,9 *$ & $>0,05$ \\
\hline Entregar consejería previo al test & 87,9 & 87,4 & 88,4 & $>0,05$ & $93,3^{*}$ & $98,1^{*}$ & 89,0 & $>0,05$ \\
\hline $\begin{array}{l}\text { Realizarse test de Elisa para ser } \\
\text { contratado }\end{array}$ & 56,0 & 49,2 & 62,1 & $>0,05$ & $70,3^{*}$ & $74,4^{*}$ & $66,6^{*}$ & $>0,05$ \\
\hline $\begin{array}{l}\text { Informar a tutores que joven de } \\
14 \text { años vive con VIH/SIDA }\end{array}$ & 11,6 & 11,1 & 11,9 & $>0,05$ & $25,5^{*}$ & $35,5^{*}$ & $16,6^{*}$ & $>0,05$ \\
\hline $\begin{array}{l}\text { Tomar test de Elisa antes de } \\
\text { cirugía, aún sin consentimiento }\end{array}$ & 54,5 & 54 & 54,9 & $>0,05$ & 70,0 * & $82,8^{*}$ & 58,4 & $>0,05$ \\
\hline $\begin{array}{l}\text { Comunicar a cónyuges resultado } \\
\text { del test }\end{array}$ & 37,8 & 34 & 41,3 & $>0,05$ & $59,3^{*}$ & $70,4^{*}$ & $49,5^{*}$ & $>0,05$ \\
\hline Realizar test de Elisa en urgencia & 43,9 & 43,9 & 43,8 & $>0,05$ & $66,2^{*}$ & $79,8^{*}$ & $54,0 *$ & $>0,05$ \\
\hline $\begin{array}{l}\text { Advertir a colega antes de } \\
\text { realizar procedimiento }\end{array}$ & 13,9 & 11,8 & 15,8 & $>0,05$ & $34,4^{*}$ & $52,1^{*}$ & $18,6^{*}$ & $>0,05$ \\
\hline Promedio global & 44,7 & 42,6 & 46,9 & $>0,05$ & $61,8^{*}$ & $71,7^{*}$ & $53,0^{*}$ & $>0,05$ \\
\hline
\end{tabular}

${ }^{*} p<0,05$ al comparar resultados inicial versus final, Test de $\chi^{2}$.

Tabla 5. Análisis de Score de la Ley de SIDA para aplicación en el escenario laboral $(\mathbf{n}=\mathbf{5 5 5})$

\begin{tabular}{|lccc|}
\hline & $\begin{array}{c}\text { Intervención } \\
\text { Promedio Score (ds) }\end{array}$ & $\begin{array}{c}\text { Control } \\
\text { Promedio Score (ds) }\end{array}$ & Ambos grupos \\
\hline Evaluación Inicial & $3,8(\mathrm{ds})$ & $4,2(\mathrm{ds})$ & 4,0 \\
Evaluación Final & $6,4(\mathrm{ds})$ & $4,8(\mathrm{ds})$ & $5,5^{*}$ \\
Valor p (evaluación inicial versus final) & $<0,001$ & $<0,05$ & $<0,001$ \\
\hline
\end{tabular}

${ }^{*} p<0,05, T$ test de comparación entre grupo intervención y grupo control. 
Conocimiento de Ley de SIDA en salud primaria - L. Ferrer et al

$\mathrm{p}<0,001)$. Dicha asociación se mantuvo independiente del nivel educacional, tipo de ocupación e ingreso económico mensual de los participantes.

\section{Discusión}

La intervención educativa demostró ser eficaz en mejorar el conocimiento de trabajadores de salud en torno a Ley de SIDA. Pertenecer al grupo intervenido se asoció en forma significativa a mayor conocimiento de esta ley, independiente de otras características sociodemográficas del grupo, como nivel educacional, tipo de ocupación e ingreso económico.

Destacó un bajo porcentaje de trabajadores que conocía la ley de SIDA, vigente desde hace cuatro años. Esta falta de conocimiento implica un conflicto no sólo para los usuarios sino también para los mismos trabajadores quienes pueden estar poniendo en riesgo su labor por no cumplir con las exigencias de la ley. El aumento de conocimiento acerca de la ley ayuda en la entrega de un cuidado que no es discriminatorio, ni estigmatizante y que protege los derechos de los trabajadores y de los usuarios, independiente de si viven o no con VIH. En Chile, este desconocimiento se asocia a otros ámbitos como son los conocimientos generales sobre VIH y uso de precauciones estándares para la prevención de $\mathrm{VIH}^{18-21}$. Todos factores que debilitan la atención relacionada con la temática de $\mathrm{VIH}^{22}$.

Estos resultados se asocian también a la percepción que tienen algunos profesionales de salud en formación. Ellos establecen que esta es limitada, pasando por falta de recepción de contenidos, dentro de los que está la ley de $\operatorname{SIDA}^{23}$.

Diseñar e implementar intervenciones educativas en este tema contribuirían a mejorar la calidad de la atención en los sistemas de salud de Chile. Esto a su vez permitiría reducir la estigmatización en torno a VIH, aún presente en los trabajadores de salud en Chile ${ }^{24,25}$. Cambio esencial para mantener la salud, el bienestar y la calidad de atención en salud que merece la población chilena.

\section{Referencias}

1. ONUSIDA [Sede Web]. AIDS epidemic update: Diciembre 2009. [acceso 13 enero de 2010]. Disponible en: http://www.unaids.org/en/KnowledgeCentre/HIVData/
EpiUpdate/EpiUpdArchive/2009/default.asp

2. Minsal (Sede web) Evolución del VIH-SIDA Chile, 19842009 [acceso 26 mayo 2011]. Disponible en: http://epi. ninsal.cl/epi/html/bolets/reportes/VIH-SIDA/Pais_8409vihSida.pdf

3. Amnistía Internacional [Sede Web]. Los derechos humanos y el VIH: mantener la promesa. Respetar, proteger y hacer realidad los derechos de todas las personas que viven con VIH/SIDA, 2007. [acceso 13 dic 2007]. Disponible en: http://web.amnesty.org/library/Index/ ESLACT750032005?open\&of=ESL-2AS - 28k -

4. Kohi T, Makone L, Chirwa M, Holzemer W, Phetlhu D, Uys L, et al. HIV and AIDS stigma violates human rights in five african countries. Nurs Ethics 2006; 13: 404-15.

5. Mesquita J, Paiva V, França I, Gravato N, Lacerda R, Della $\mathrm{M}$, et al. Vulnerability, human rights, and comprehensive health care needs of young people living with HIV/AIDS. Am J Public Health 2006; 96: 1001-6.

6. ONU [Sede Web]. Declaración universal de los derechos humanos, c1948. [acceso 17 dic 2006]. Disponible en: http://www.un.org/spanish/aboutun/hrights.htm

7. Meel B. Ethical issues related to HIV/AIDS: case reports. J Clin Forensic Med 2005; 12: 149-52.

8. ONU [Sede Web]. Declaración de compromiso en la lucha contra el VIH/SIDA, c2001. [Citado 13 dic 2007]. Disponible en: http://www.un.org/spanish/ag/sida/ aress262.pdf

9. CEPAL [Sede Web]. VIH,SIDA y derechos humanos: retos de política y legislación. c2006. [acceso 13 dic 2006]. Disponible en: http://www.eclac.org/celade/noticias/ paginas/6/27116/BadillaAE.pdf

10. Deliyanis V. Ley de SIDA en Chile: un sueño hecho realidad. Vivo Positivo 2002; 4: 14-5.

11. Establece normas relativas al virus de inmuno deficiencia humana y crea bonificación fiscal para enfermedades catastróficas. Ley 19.779 de 4 de diciembre. Diario Oficial de La República de Chile (14.12.2001).

12. Herramientas para el ejercicio de los derechos ciudadanos de las personas viviendo con VIH/SIDA [Sede Web]. Santiago: Vivo Positivo; c2004. [acceso 13 dic 2006]. Disponible en: http://www.vivopositivo.org/portal/datos/ $\mathrm{ftp} /$ Herramientasderechosciudadanos.doc

13. Vidal VF, Carrasco SM, Santana NP. Discriminación por VIH/SIDA en Chile: cambios y continuidades 2002 2005. Santiago: FLACSO, 2007.

14. Kinsler J, Wong M, Sayles J, Davis C, Cunningham W. The effect of perceived stigma from a health care provider on access to care among a low-income HIV-positive population. AIDS Patient Care STDS 2007; 21: 584-92.

15. Norr K. Mobilizing Health Workers for Community HIV prevention in Chile. Grant \#1 R03 TW006980-02, 
2004-2007. [Formulario de aplicación].

16. OMS. The world health report 2006 - working together for health. 2006. Recuperado de [http://www.who.int/ whr/2006/en/index.html] en junio de 2008.

17. Norr K. Mobilizing Health Workers for Community HIV prevention in Malawi, África. Grant NR08058, 20012006. [Formulario de aplicación].

18. Ferrer L, Cianelli R, Norr K, Cabieses B, Araya A, Bernales $\mathrm{M}$, et al. Observed use of standard precautions in Chilean community clinics. Public Health Nursing 2009; 26: 440-8.

19. Ferrer L, Cianelli R, Bernales M. VIH y SIDA en Chile: Desafío para su prevención. Temas De La Agenda Pública-UC 2009; 4: 1-11.

20. Ferrer L, Cianelli R, Guzmán E, Cabieses B, Irarrázabal L, Bernales M, et al. Chilean university students: Knowledge and concern about HIV/AIDS. Janac-Journal Of The Association Of Nurses In Aids Care 2007; 18: 51-6.

21. Herrera A, Cianelli R, Ferrer L. Estigma y Discrimina- ción a Usuarios Con VIH/SIDA por Parte de los Trabajadores de la Salud. Revista Horizonte de Enfermería 2005; 16: 49-58.

22. Ferrer L, Michele I, Cianelli R. Stories From Santiago: HIV/AIDS and Needed Health Systems Change. Advances In Health Care Management 2005; 5: 31-72.

23. Ferrer L, Bernales M, Cabieses B, Cianelli R, Triviño $\mathrm{X}$, Irarrázabal L, et al. Conocimientos y percepción de formación curricular asociada al VIH/SIDA de futuros enfermeros y médicos en Santiago. Revista Horizonte De Enfermería 2007; 18: 53-9.

24. Cianelli R, Ferrer L, Norr K, Irarrázabal L, Bernales M, Miner S, et al. Stigma related to HIV among community health workers in Chile. Stigma Research and Action Journal 2011; 1: 3-10.

25. Norr K, Ferrer L, Cianelli R, Crittenden K, Irarrázal, L, Norr J, et al (under review). Peer Group Intervention for HIV Prevention among Health Workers in Chile. Association of Nurses in AIDS Care JANAC (In press). 